\title{
Stellar populations in the dwarf galaxy NGC59
}

\author{
A.E. Sansom*, J.J. Thirlwall \\ Jeremiah Horrocks Institute, University of Central Lancashire, Preston, UK \\ E-mail: AESansom@uclan.ac.uk, JJThirlwall@uclan.ac.uk
}

P. Väisänen, A.Y. Kniazev

South African Astronomical Observatory and Southern African Large Telescope, PO Box 9 Observatory, 7935, South Africa

E-mail: petri@saao.ac.za, akniazev@saao.ac.za

\begin{abstract}
NGC59 is a low-luminosity, early-type galaxy (ETG). It is a member of the Sculptor galaxy group at a distance of $4.4 \mathrm{Mpc}$. We study the stellar population properties using data from the Robert Stobie Spectrograph (RSS) on SALT. A metallicity of $[\mathrm{Z} / \mathrm{H}] \sim-1.1$ to -1.6 was found for this galaxy, which is very low compared to similar mass galaxies. A range of ages were found in fitting the optical spectra with composite models. The spectra show emission lines from current star formation, plus strong Balmer absorption lines indicative of intermediate age stars. Fits to spectral line strengths also indicate the presence of older stars. NGC59 is an example of downsizing occurring in galaxy evolution in the local Universe.
\end{abstract}

SALT Science Conference 2015 -SSC2015-

1-5 June, 2015

Stellenbosch Institute of Advanced Study, South Africa

${ }^{*}$ Speaker. 


\section{Introduction}

Massive elliptical galaxies are thought to be an end state of galaxy evolution, built up by hierarchical mergers of lower mass systems. This lower mass progenitor population will not all have merged by the present day. Here we investigate the stellar populations of a nearby, earlytype (dS0) galaxy NGC59, which may represent a relic left over from the early formation era. Its velocity dispersion is only $\sigma=37 \mathrm{~km} \mathrm{~s}^{-1}$, making it one of the lowest mass ETGs to be studied for its composite stellar populations. Star formation is still taking place within NGC59. We fit single age, single metallicity stellar population (SSP) and composite star formation history (SFH) models to find out about the properties of the bulk of the stars. RSS, long-slit spectra in the blue (PG3000 grating) and red (PG1300 grating) regions are combined to give wavelength coverage from $3500-6100 \AA$.

\section{Lick indices and analysis}

Emission line contributions were removed and Lick indices were measured in three regions across the galaxy, as described in [5]. These are shown plotted against grids of model predictions in Figure 1. From chi-squared fitting to Lick indices, a luminosity weighted mean central age of $\sim 5$ Gyr was derived, with a mean $[\mathrm{Fe} / \mathrm{H}] \sim-1.4$ (i.e. $<1 / 25$ th solar metallicity). Low mean metallicities were also found further from the centre of the galaxy. Ages are difficult to constrain at these low metallicities, as illustrated by the sharp turn-up of the grids in Figure 1a at low values of Fe5335.

Figure $1 \mathrm{~b}$ illustrates how indices behave for different abundance patterns. The Mgb index is sensitive to magnesium whilst Fe5335 is sensitive to iron abundance. This figure shows that at low metal line strengths, corresponding to low metallicities, the grids of model predictions merge, making it difficult to distinguish an $\alpha$-enhanced stellar population from one that has a scaled-solar abundance pattern.

Fits of multiple SSPs indicated that there are both young and old stars in this galaxy. This can be seen directly from the spectra in which hydrogen lines are seen in absorption up to at least the 9th line in the Balmer series, due to the efficient blue response of the SALT telescope [4].

\section{The mass-metallicity relation}

In Figure 2 we have compared the metallicity ([Fe/H]) in NGC59 with similar mass galaxies, showing mean trends from a number of studies. Stellar velocity dispersion is used as a proxy for stellar mass in this plot. Comparison data from [3] (hereafter K11, their figure 13) cover the most relevant range of $\sigma$, wherease most studies of ETGs cover high $\sigma$ regions, but do not cover this low $\sigma$ region very well (e.g. [1], [2]). The metallicity in NGC59 is very low. It is an outlier in the distribution of observed metallicities for galaxies at this velocity dispersion, even taking into account the increased scatter of points at lower $\sigma$. Only dwarf spheroidals at lower $\sigma$ from K11 show similarly low metallicities to NGC59 (see the four vertical green lines in Figure 2). More examples of galaxies with low velocity dispersions are needed to see how the mass-metallicity relation behaves for galaxies in this relatively unexplored region of parameter space. 

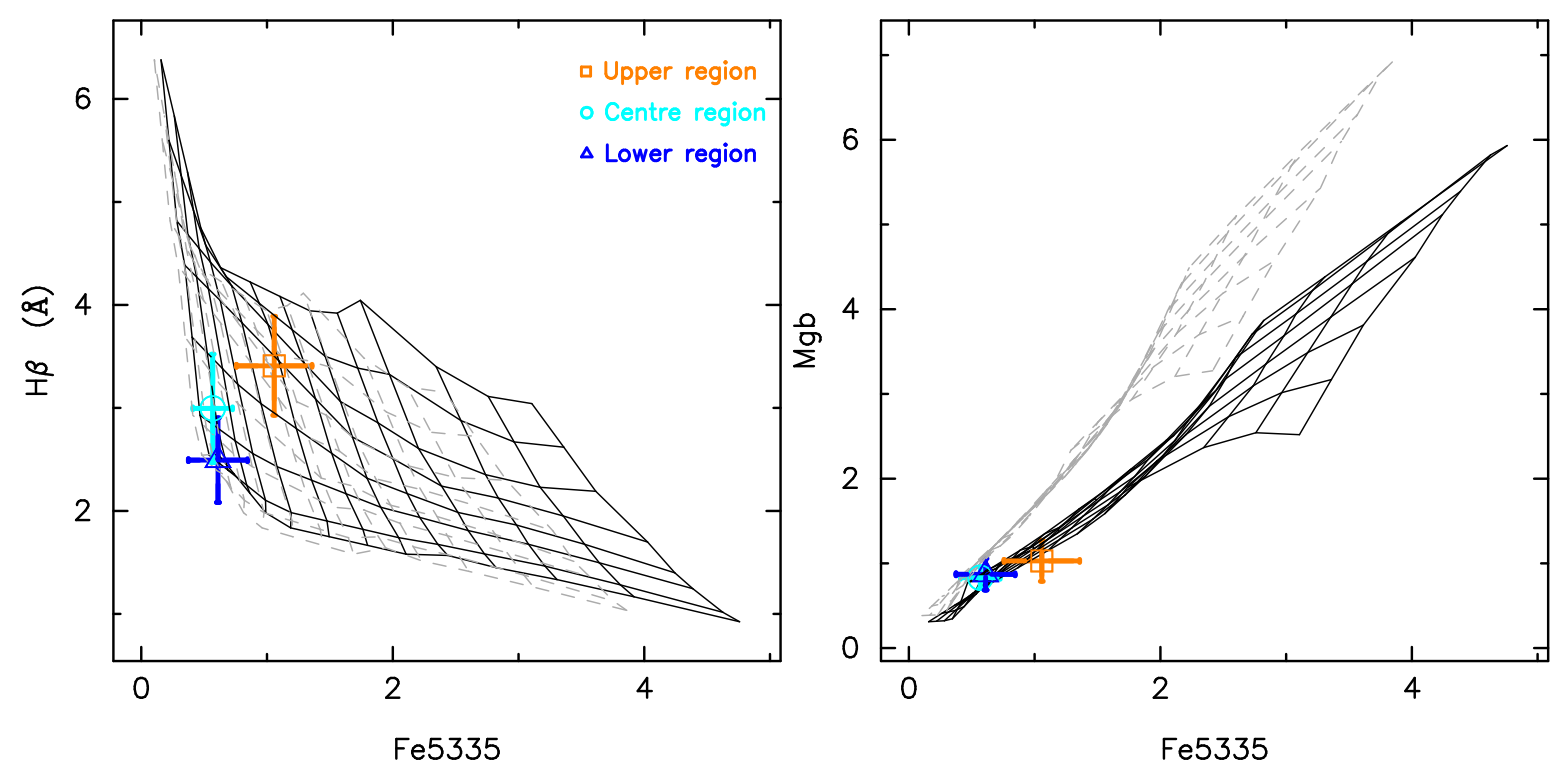

Figure 1: Lick indices plotted against SSP grids of models from the MILES stellar library. Black grid shows $[\alpha / \mathrm{Fe}]=0$, grey grid shows $[\alpha / \mathrm{Fe}]=+0.3$. The left plot highlights the weak Fe line strengths, whilst the right plot shows how difficult it is to determine element abundances at low Fe abundance. Details of these fits are described in [5].

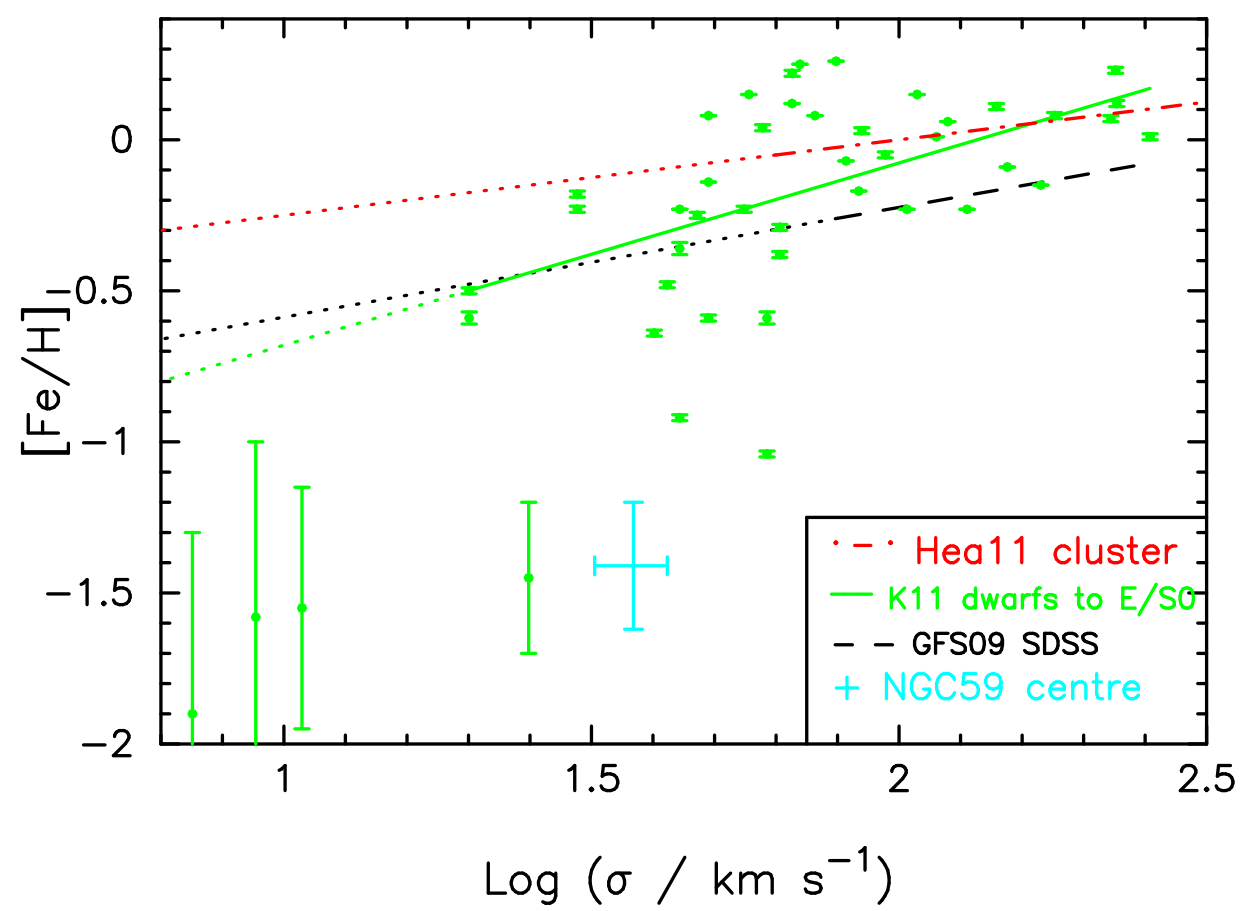

Figure 2: Plots of mass-metallicity relations in ETG samples, with stellar velocity dispersion $\sigma$ as a proxy for mass and $[\mathrm{Fe} / \mathrm{H}]$ on the vertical axis. The extensive study of [3] (K11 in green) includes dSphs (resolved populations) and unresolved studies of ETGs with a wide range of stellar velocity dispersions, with their mean trend shown here as a solid green line, plus the points from their figure 13. Mean trends from other authors are also shown ([1] in black, [2] in red), with dotted regions indicating extrapolations of mean trends fitted to higher $\sigma$ galaxies. The plot shows results for NGC59 against these mean trends from several authors, revealing NGC59 as an outlier with unexpectedly low stellar metallicity ([5] in turquise). 


\section{Conclusion}

The low-luminosity ETG NGC59 in the Sculptor group was observed over a wide optical spectral range with SALT. Fitting SSPs, we find evidence for extremely low metallicity in its centre, plus young and old stellar population components from the presence of abosorption and emission lines (see [5] for further details). Regions further out also show a wide range of ages and stars with significantly lower metallicity than the gas. The evolution of this galaxy merits further study and modelling. It is an outlier in the mass-metallicity relation.

\section{References}

[1] G.J. Graves, S.M. Faber, R.P. Schiavon, Dissecting the Red Sequence. I. Star-Formation Histories of Quiescent Galaxies: The Color-Magnitude versus the Color-sigma Relation, ApJ 693 (2009), 486.

[2] C.D. Harrison et al., The stellar populations of early-type galaxies - II. The effects of environment and mass, MNRAS, 413 (2011), 1036.

[3] M. Koleva, P. Prugniel, S. De Rijcke, W. Zelinger, Age and metallicity gradients in early-type galaxies: a dwarf-to-giant sequence, MNRAS, 417 (2011), 1643.

[4] D. O'Donoghue et al., First science with the Southern African Large Telescope: peering at the accreting polar caps of the eclipsing polar SDSS J015543.40+002807.2, MNRAS, 372 (2006), 1510.

[5] A.E. Sansom, J.J. Thirlwall, M.A. Deakin, P. Väisänen, A.Y. Kniazev, J. Th. van Loon, The stellar populations in the low-luminosity, early-type galaxy NGC 59, MNRAS, 450 (2015), 1338. 